\title{
EXTRAGALACTIC RADIO SOURCES
}

Edited by R. EKERS, C. FANTI and L. PADRIELLI
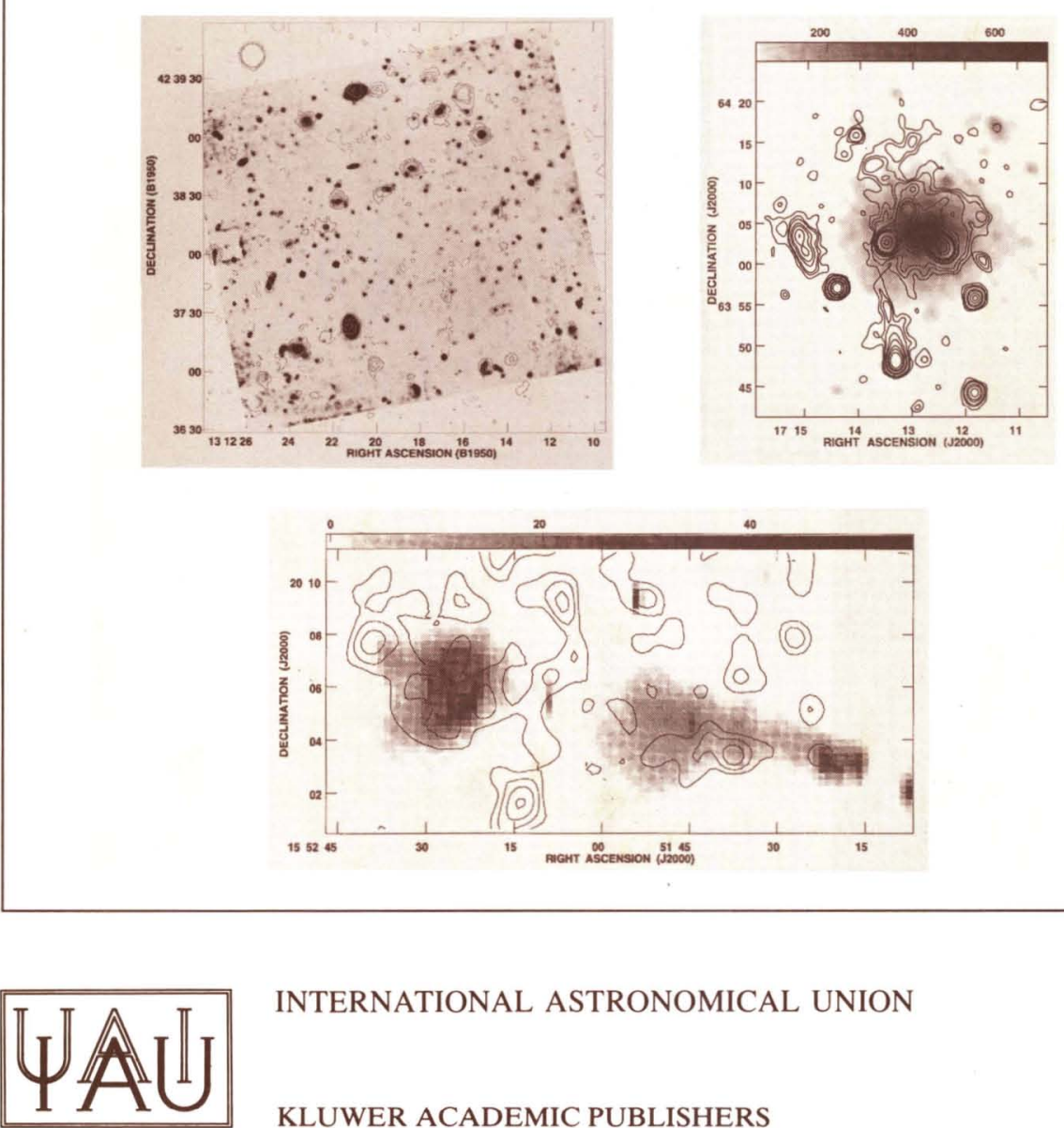

INTERNATIONAL ASTRONOMICAL UNION

KLUWER ACADEMIC PUBLISHERS 
EXTRAGALACTIC RADIO SOURCES 


\title{
EXTRAGALACTIC RADIO SOURCES
}

PROCEEDINGS OF THE 175TH SYMPOSIUM OF THE INTERNATIONAL ASTRONOMICAL UNION, HELD IN BOLOGNA, ITALY

10-14 OCTOBER 1995

\author{
EDITED BY \\ R. EKERS \\ Australia Telescope, CSIRO, \\ Epping, Australia \\ C. FANTI \\ Istituto di Radioastronomia, \\ CNR, Bologna, Italy \\ and

\section{PADRIELLI} \\ Istituto di Radioastronomia, \\ CNR, Bologna, Italy
}

\section{KLUWER ACADEMIC PUBLISHERS}

DORDRECHT / BOSTON / LONDON

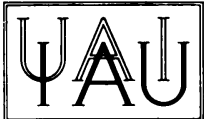




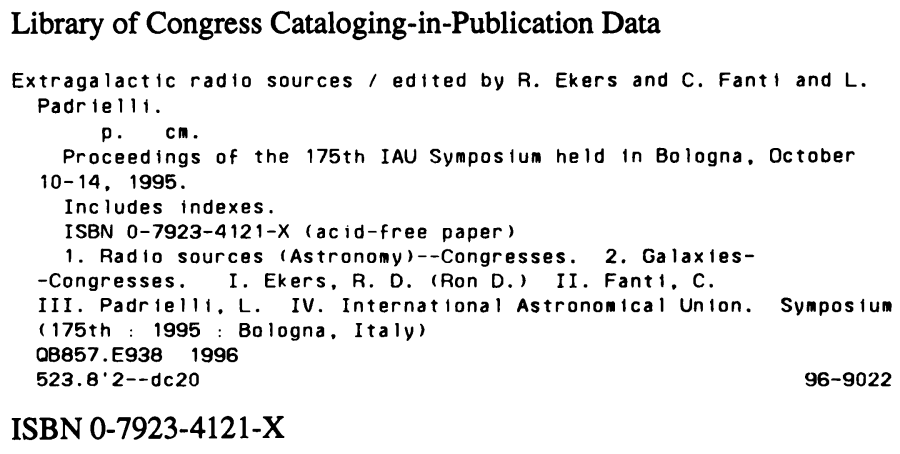

Published on behalf of

the International Astronomical Union

by

Kluwer Academic Publishers, P.O. Box 17, 3300 AA Dordrecht, The Netherlands.

Kluwer Academic Publishers incorporates the publishing programmes of

D. Reidel, Martinus Nijhoff, Dr W. Junk and MTP Press.

Sold and distributed in the U.S.A. and Canada

by Kluwer Academic Publishers,

101 Philip Drive, Norwell, MA 02061, U.S.A.

In all other countries, sold and distributed

by Kluwer Academic Publishers Group,

P.O. Box 322, 3300 AH Dordrecht, The Netherlands.

Printed on acid-free paper

\author{
All Rights Reserved \\ C1996 International Astronomical Union
}

No part of the material protected by this copyright notice may be reproduced or utilized in any form or by any means, electronic or mechanical including photocopying, recording or by any information storage and retrieval system, without written permission from the publisher.

Printed in the Netherlands 


\section{TABLE OF CONTENTS}

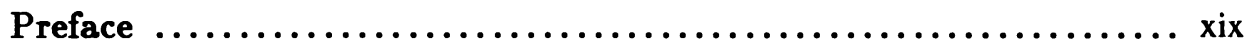

Organizing Committees $\ldots \ldots \ldots \ldots \ldots \ldots \ldots \ldots \ldots \ldots \ldots \ldots \ldots \ldots \ldots \ldots \ldots \ldots \ldots$

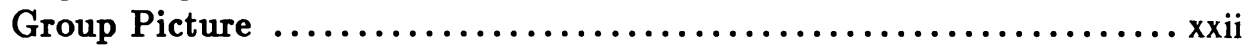

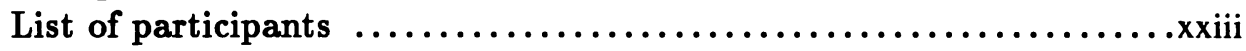

Guglielmo Marconi and Radioastronomy (Invited) $\ldots \ldots \ldots \ldots \ldots \ldots \ldots 1$ G.Setti

\section{Properties of Radio Sources}

pc-scale Structure of Compact Radio Sources (Review) $\ldots \ldots \ldots \ldots \ldots . \ldots 5$ J.A. Zensus

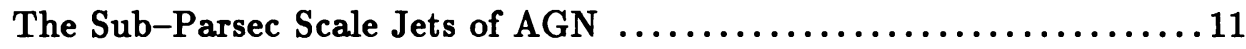

T.P. Krichbaum, W. Alef, A. Witzel

Radio Observations of the $\gamma$-Ray Blazar 0528+134 ............. 14

M. Pohl, W. Reich, T.P. Krichbaum, et al.

A Dramatic (and Invisible!) flare in NRAO530

G.C. Bower, D. Backer, R. Forster, M. Wright

VLBI Observations of Southern EGRET Identifications

J.E.J. Lovell, S.J. Tingay, P.G. Edwards, et al.

Monitoring the Jet in Centaurus A at 0.1 pc Resolution

R.A. Preston, S.J. Tingay, D.L. Jauncey, et al.

A Counterjet in the Nucleus of Centaurus A ...................23

D.L. Jones, S.J. Tingay, R.A. Preston, et al.

High frequency radio observations of the nucleus Centaurus $A \ldots \ldots 25$ Z. Abraham

Simultaneous multi-frequency imaging of the nucleus of NGC $1275 \ldots 27$

J.D. Romney, R.C. Walker, K.I. Kellermann, et al.

Constraints on the accretion region in NGC1275 from

VLBA observations of the counterjet ..................... 30

R.C. Walker, J.D. Romney, R.C. Vermeulen, et al.

Continuum VLBI Polarimetry of $3 \mathrm{C} 454.3$ at $43 \mathrm{GHz}$ .33

A.J. Kemball, P.J. Diamond

Multi-Frequency VLBI observations of $3 \mathrm{C} 390.3 \ldots \ldots \ldots \ldots \ldots \ldots \ldots . \ldots . \ldots . \ldots 55$

E. Preuss, W. Alef, K.I. Kellermann 


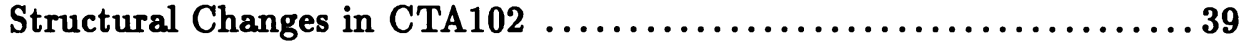

F.T. Rantakyrö, L.B. Båath

VLBA Studies of BL Lac

G. Denn, R. Mutel

Helical Motion in the BL LAC Object OJ287?

L. Vicente, P. Charlot, H. Sol

Variability characteristics of Blazar OJ287

L.O. Takalo, A. Sillanpää, T. Pursimo, et al.

The Environment of OJ287:

Nearby Galaxies and a Long Optical Jet?

A. Sillanpää, L. Takalo, K. Nilsson, et al.

New Results from VLBI Polarization Observations

of BL Lacertae Objects

D.C. Gabuzda

$3.6 \mathrm{~cm}$ VLBI Total Intensity and Polarization Images

of BL Lacertae Objects ................................ 51

D.C. Gabuzda, A.B. Pushkarev, T.V. Cawthorne

Extended Emission in BL Lac Objects

M. Bondi, D. Dallacasa, C. Stanghellini, R. Della Ceca

Arcsecond Scale Polarization of BL Lac Objects

C. Stanghellini, P. Cassaro, M. Bondi, et al.

Superluminal motions: the first 100 sources (Review) $\ldots \ldots \ldots \ldots \ldots 57$ R.C. Vermeulen

CSS versus Large Size Sources (Invited) $\ldots \ldots \ldots \ldots \ldots \ldots \ldots \ldots \ldots \ldots 6 . \ldots \ldots$

R. Fanti, R.E. Spencer

A VLBI Study of GHz-Peaked-Spectrum Radio Sources

C. Stanghellini, C.P. O'Dea, S.A. Baum, et al.

2.23 and $8.29 \mathrm{GHz}$ Observations of CSS-GPS Candidates

D. Dallacasa, M. Bondi, F. Mantovani

Large Bent Jets in the Inner Region of CSS

F. Mantovani, W. Junor, M. Bondi, et al.

High Resolution Structure of Southern

Compact Steep Spectrum Sources .........................73

A. Tzioumis, R. Morganti, C. Tadhunter, et al.

A Single Baseline VLBI Survey of

Southern Peaked Spectrum Sources ....................... 75

E.A. King, P.M. McCulloch, D.L. Jauncey, et al.

Extreme Depolarization in Compact Steep Spectrum sources .........77

S.T. Garrington, C.E. Akujor

Large Rotation Measures in CSS Sources

W. Junor, F. Mantovani, A. Peck, et al. 
NLR Kinematics in CSS Radio Sources

R. Gelderman

A World Array VLBI Image of $3 \mathrm{C} 216$ at $1.6 \mathrm{GHz}$

C.E. Akujor, R.W. Porcas, I. Fejes

$5 \mathrm{GHz}$ EVN Polarization of $3 \mathrm{C} 286$

D. Dallacasa, R.T. Schilizzi, H.S. Sanghera, et al.

The Evolution of Extragalactic Radio Sources

A.C.S. Readhead, T.J. Pearson, G.B. Taylor, P.N. Wilkinson

PKS 1413+135: A Very Young Radio Galaxy

E.S. Perlman, C.L. Carilli, J.T. Stocke, J. Conway

A Circumnuclear HI Disk in the Compact Symmetric

Object 4C31.04?

J.E. Conway

Multifrequency Study of VLBI-Compact Sources

1-22 Ghz Spectra Observations and Model Fits ..............95

Yu.A. Kovalev, A.B. Berlin, N.A. Nizelskij, et al.

Statistical Analysis of Observations of a Complete Sample

of Compact Extragalactic Radio Sources .................. 97

Y.Y. Kovalev

High Radio Frequency Studies of Equatorial and Southern AGN .....99

M. Tornikoski, E. Valtaoja

A Simple Method to Analyze Radio Frequency Outbursts of

Quasars ............................................ 101

H. Teräsranta, E. Valtaoja, M. Lainela

Long-term Variability of Extragalactic Radio Sources $843 \mathrm{MHz} \ldots \ldots 103$

R.W. Hunstead, B.M. Gaensler

Gravitational lenses (Invited)

J.N. Hewitt

Scintillating Lenses

M.A. Walker

A VLA Study of Ooty Small Diameter Sources

T.K. Menon

Multi-frequency VLBI Observations of

the Gravitational Lens B0218+357

R.W. Porcas, A.R. Patnaik

PKS 1117+146: Gravitational Lens or Micro-lobes

M. Bondi, M. Garrett, L. Gurvits

High Precision Astrometry over Large Angular Scales with Closure

Constraints: the Triplet $1803+784 / 1928+738 / 2007+777 \ldots \ldots \ldots 120$

E. Ros, J.M. Marcaide, J.C. Guirado, et al.

EVN Phase Referenced Observations of $1308+328$

M.J. Rioja, R.W. Porcas, J. Machalski 
viii

VLBI Observations of FRI Radio Galaxies .....................124

T. Venturi, W.D. Cotton, L. Feretti, et al.

Parsec Scale Properties of Low Power Radio Galaxies

G. Giovannini, W.D. Cotton, L. Lara, T. Venturi

VLBI Observations of Low-Redshift Radio Galaxies ..............129

S.J. Tingay, D.L. Jauncey, R.A. Preston, et al.

The relativistic Jet in M84 .................................131

J.M. Wrobel, R.C. Walker, A.H. Bridle

The parsec-scale nucleus and jets of Hydra A

G.B. Taylor

Low Luminosity Radio Galaxies (Review)

P. Parma, H.R. de Ruiter, R. Fanti

FR I Jets in Southern Radio Galaxies

P.A. Jones, B.D. Lloyd

High-Frequency Study of Low and Intermediate Luminosity

Radio Galaxies

L. Gregorini, P. Parma, U. Klein, K.-H. Mack

Large Scale Structure: Jets on kiloparsec Scales (Review)

R.A. Laing

The Jets in FRII Radio Galaxies with $\mathrm{z}<0.3 \ldots \ldots \ldots \ldots \ldots \ldots . \ldots 153$

M. Hardcastle

Low-Luminosity Radio QSOs: the B2 sample .................155

J.I. González-Serrano, I. Pérez-Fournon, L. Gregorini, et al.

An Atlas of Extragalactic Radio Sources ......................157

J.P. Leahy, A.H. Bridle, R.G. Strom

Extreme Rotation Measure Radio Galaxies (Invited) .............159

C.L. Carilli, R.A. Perley, G. Miley, et al.

Radio Polarisation Studies of Galaxies at $z>2 \ldots \ldots \ldots \ldots \ldots \ldots . \ldots 163$

R.M. Athreya, V.K. Kapahi, P.J. McCarthy, W. van Breugel

Cygnus A: Some Topics from the Workshop (Review) .............167

D.E. Harris

First millimeter Mapping of the jet and nucleus of M87 ...........175

V. Despringre, D. Fraix-Burnet

Unusual Radio Structures in Markarian 6

M.J. Kukula, A. Pedlar, S.A. Baum, A.J. Holloway

IRAS 0421+0400: A Curious Spiral with Flaring Jets

W. Steffen, A.J. Holloway, A. Pedlar, D.J. Axon

Analysis of VLBI Spot Map of $\mathrm{H}_{2} \mathrm{O}$ Masers for the

HII Region Complex IC133 of Spiral Galaxy M33

Yu Zhi-yao

A Search for HI in Strong Radio Galaxies

S.M. Simkin, J. Callcut 
A Multi-radio-frequency Study of a RQQ $\ldots \ldots \ldots \ldots \ldots \ldots \ldots \ldots \ldots \ldots \ldots$ K.M. Blundell, M. Lacy

Discussion

\section{Relation between Radio and Other Wavelenghts}

Hubble Space Telescope Observations of Extragalactic Jets (Review) .195 F.D. Macchetto

HST Observations of Jets and Radio Lobes ...................201

$P$. Crane

Merlin and HST Observations of the Jet in 3C273 203

R.C. Conway, S.T. Garrington, T.W.B. Muxlow, R.J. Davies

Circumnuclear Disks in Radio-Quiet Active Galaxies (Review) ......205 A.S. Wilson

Probing AGN with Water Masers (Invited)

J.M. Moran, L.J. Greenhill, J.R. Herrnstein, et al

What Powers Ultra-Luminous IRAS Galaxies?

R.B. Partridge, J. Marr, T. Crawford, M. Strauss

Optical-Radio Connections (Review)

C.N. Tadhunter

Optical Polarization of 3C 265

M.H. Cohen, H.D. Tran, P.M. Ogle, R.W. Goodrich

A Powerful Jet/Cloud Interaction in the Radio Galaxy

PKS 2250-41 (Invited)

R. Morganti, C.N. Tadhunter, N. Clark, N. Killeen

Radio Outflows and the Origin of the Narrow Line Region

in Seyfert Galaxies

A. Capetti, D.J. Axon, F.D. Macchetto, W.B. Sparks

Matter-Bounded Photoionized Clouds

L. Binette, A.S. Wilson, T. Storchi-Bergmann

Is the UV Alignment Effect Present in Low Redshift Radio Galaxies? 234 A. Cimatti, S. di Serego Alighieri

Weak Radio Galaxies: Narrow-Band Optical Imaging .236

R. Carrillo, I. Cruz-González

Optical Properties of FRI Radio Galaxies 238

M.J. Ledlow, F.N. Owen

Optical Properties of B2 Radio Galaxies

J.I. González-Serrano, R. Carballo

Nearly Simultaneous Optical and VLBI Polarization

Observations of BL Lacertae Objects ......................242

D.C. Gabuzda, P.Y. Kochanev, M.L. Sitko, P.S. Smith 
Can BL Lac Nuclei and Broad Line Regions Coexist? or When is an Object a BL Lac? ............................ 244 M.J.M. Marchã

Intraday Variability Statistics of Radio-Selected and X-Ray

Selected BL Lac Objects ..............................246

J. Heidt

Host Galaxies of Radio-Loud \& Radio-Quiet AGN .............. 248

M.J. Kukula, J.S. Dunlop, G.L. Taylor, D.H. Hughes

A Possible Fundamental Difference Between

Radio Loud and Radio Quiet AGN ......................250

M. Calvani, J.W. Sulentic, P. Marziani, et al.

The Radio and Optical Properties of Quasars

C.D. Impey, E.J. Hooper, C.B. Foltz, P.C. Hewett

Optical Properties of the Radio Source B2 0828+32

254

M.H. Ulrich-Demoulin, J. Rönnback

Inverse Compton X-Rays from Giant Radio Galaxies

D. Tsakiris, J.P. Leahy, R.G. Strom, C.R. Barber

ROSAT Studies of Extragalactic Radio Sources (Invited)

W. Brinkmann, J. Siebert, W. Yuan

Soft X-Ray Properties of a Complete Sample of Radio Sources

$J$. Siebert, W. Brinkmann, R. Morganti, et al.

X-Ray Spectra of BL Lacertae Objects from the ROSAT Archive ....265

G. Lamer, H. Brunner, R. Staubert

The Rosat X-Ray Spectra of BL Lacertae Objects

P. Padovani, P. Giommi

The Search for a New BL Lac Sample

A. Wolter, A. Caccianiga, T. Maccacaro, C. Ruscica

Optical Identifications for a Sample of REXs:

Search for BL Lac Objects

C. Ruscica, A. Caccianiga, T. Maccacaro, A. Wolter

X-Ray Spectral Properties of Radio Galaxies (Invited) ............273

H. Inoue

Gamma-Ray Emission from Extragalactic Radio Sources:

EGRET Observations of Active Galactic Nuclei (Invited) .......277

P.F. Michelson

The Big Picture from Radio Waves to Gamma Rays .............281

C.D. Impey

The Radio-Gamma-Ray Connection: the Radio Properties

of Gamma-Ray-Bright Blazars

M.F. Aller, H.D. Aller, P.A. Hughes 
AGN Statistics of Simultaneous Radio and

Gamma Ray Observations ............................ 285

A. Mücke, M. Pohl, G. Kanbach, et al.

Optical Monitoring of Gamma-Ray Loud Blazars

C.M. Raiteri, G. Ghisellini, M. Villata, et al.

Optical Monitoring of the Gamma-Ray Loud

BL Lac Object S5 0716+714

G. Latini, S. Bosio, G. De Francesco, et al.

The Highest Energy Cosmic Rays (Review)

C.A. Norman

Discussion

Radio Sources and their Environment

Radio Sources in Clusters of Galaxies (Review) ................. 305

F.N. Owen

General Properties of Giant Radio Galaxies

U. Klein, K.H. Mack, L. Saripalli

Morphologies in Mpc-Size Radio Galaxies ...................... 313

L. Saripalli, R. Subrahmanyan, R.W. Hunstead

Towards a Complete Sample of Giant Radio Galaxies at $\mathrm{z}>0.4 \ldots \ldots .315$

A.P. Schoenmakers, H. Röttgering, H. van der Laan, et al.

Spectral Indices and Particle Ageing Studies on

the Giant Radio Galaxy NGC6251

K.H. Mack, U. Klein, L. Saripalli, et al.

Evolution of Powerful Extended Radio Sources R.A. Daly

Radio Source Environment at Redshift $>0.5$

M. Lacy, S. Rawlings, M. Wold, et al.

The Environments of Radio Galaxies

J.E. Pesce, R. Falomo, G. Fasano, R. Scarpa

Clusters of Galaxies at Intermediate Redshifts:

a Sample Selected at Radio Wavelengths

A.Zanichelli, R. Scaramella, M. Vigotti, et al.

Quasar Radio Morphology and Environment at $\mathrm{z} \sim 1 / 2$

T.A. Rector, J. Stocke, E. Ellingson

Quasar Radio Structure in Cluster Environments

J.B. Hutchings, A.C. Gower, S. Ryneveld, A. Dewey

Radio-Optical Alignments in Nearby

Cooling Flow Cluster Central Galaxies

B.R. Mcnamara 
Diffuse Cluster Radio Sources (Review) $\ldots \ldots \ldots \ldots \ldots \ldots \ldots \ldots \ldots \ldots . \ldots . \ldots 33$

L. Feretti, G. Giovannini

Electron Transport in the Coma Cluster

J.G. Kirk, P. Duffy, R.O. Dendy

Coma Cluster: CR Acceleration and Gamma-Ray Production

A.V. Dogiel

The Radio Lobes of Virgo A

H. Rottmann, K.H. Mack, U. Klein, et al.

The Relic Radio Source B2 0924+30

U. Klein, K.H. Mack, L. Gregorini, P. Parma

Radio Spectra and Particle Ages of the Head-Tail

Radio Galaxy NGC1265

L. Feretti, G. Giovannini, U. Klein, et al.

Observations of Straight-angle Tailed Radio Galaxies in

Rich Clusters of Galaxies

A.A. O'donoghue, J.A. Eilek, F.N. Owen

The Environment of Hercules A

N.A.B. Gizani, J.P. Leahy

Multi-Wavelenght Study of Rosat Clusters of Galaxies

A.D. Reid, R.W. Hunstead, M.M. Pierre

ATCA 13 and $20 \mathrm{~cm}$ Observations of A3556

S. Bardelli, R. Morganti, T. Venturi, R.W. Hunstead

The X-ray Intracluster Medium (Invited)

H. Böhringer

A2241: Clusters with Head-Tails at X-Rays

L. Norci, L. Feretti, E.J.A. Meurs

ASCA observations of clusters of galaxies (Invited)

K. Yamashita

Discussion

\section{Unification Issues}

Unified Schemes for Extragalactic Radio Sources (Review) .........373 Gopal-Krishna

The Unification of Radio-Loud AGN .........................379

C.M. Urry, P. Padovani

Orientation Effects and Seyfert Cores (Invited) $\ldots \ldots \ldots \ldots \ldots \ldots \ldots . \ldots 381$

R.P. Norris, A.L. Roy

New Observations of BL Lacertae Objects (Invited) $\ldots \ldots \ldots \ldots \ldots \ldots 385$

J.T. Stocke

The Unification of Quasars and Radio Galaxies .389

S. di Serego Alighieri 
The Evolutionary Unified Scheme, Jet Asymmetry, and

Superluminal Motion .........................................

F. Vagnetti

Radio Structures of the MRC 1-Jy Sources and the Unification

of Radio Galaxies and Quasars .............................393

V.K. Kapahi, R.M. Athreya, C.R. Subrahmanya, et al.

Spectral Index Asymmetries in Low-z Radio Galaxies ............ 395

J. Dennett-Thorpe, A.H. Bridle, R.A. Laing, P.A.G. Scheuer

Depolarization Studies of Radio Sources and the Unified Scheme .....397

S.T. Garrington, G.F. Holmes, D.J. Saikia

The UV/Radio Correlation of Quasars and its Implications

for Unified Schemes ....................................400

H. Falcke

KECK Spectropolarimetry of High Redshift Radio Galaxies ....... 402

A. Cimatti, A. Dey, W. van Breugel, et al.

Composite Optical Spectra of Radio Quasars ..................405

J.C. Baker, R.W. Hunstead

On the X-Ray Emission from the Powerful Radiogalaxies $\ldots \ldots \ldots \ldots . . .407$

G. Brunetti, G. Setti, A. Comastri

Discussion

Radio Source Modelling and Emission Mechanisms

X-Ray and Gamma-Ray Emission in Blazars (Invited) ...........413

G. Ghisellini

Cooling-Flow Models of the X-Ray Emission and

Temperature Profiles of Elliptical Galaxies ..................4 417

G. Bertin, T. Toniazzo

High-Energy Gamma and Radio Variability of Blazars

in the Model of Non-Stationary Jet

M.M. Romanova, R.V.E. Lovelace

Comptonization as Blazar High Energy Emission

O. Dreissigacker

The Nature of Flat Spectrum Sources (Invited)

D.B. Melrose

Energy Transport in Radio Galaxies and Quasars:

A Workshop Theory Review (Review)

P.E. Hardee

The Production of Jets from Magnetized Accretion Disks:

Simulation of the Blandford-Payne Mechanism

D.L. Meier, D.G. Payne, K.R. Lind 
A Numerical Study of Relativistic Jets ..................... 435

J.A. Font, J.M. Marti, J.M. Ibáñez, E. Müller

Viewing Angle and the Appearance of Superluminal Jets .......... 437

H.D. Aller, M.F. Aller, P.A. Hughes, A. Mioduszewski

Simulations of Superluminal Radio Sources ................... 439

S.S. Komissarov, S.A.E.G. Falle

Physics of the M87 Jet ...................................441

M.C. Begelman, G.V. Bicknell

Relativistic Electron-Positron Clouds in VLBI Jets ..............443

V. Despringre, D. Fraix-Burnet

The Cosmic Nozzle

M.V. Konjukov

Intrinsic Kinematics of Moving Components in

Curved Relativistic Jets

L. Lara, A. Alberdi

Supersonic Jets and Lobe Morphologies (Invited)

A. Ferrari, S. Massaglia, G. Bodo

3-D Simulations of Kelvin-Helmholtz Instabilities in

Supersonic Jets

P. Rossi, G. Bodo, S. Massaglia, et al.

MHD Self-Similar Solutions for Collimated Jets

E. Trussoni, C. Sauty, K. Tsinganos

MHD equilibria of Helical Jets

M. Villata, A. Ferrari

A New Analytical Jet Model

C.R. Kaiser, P. Alexander

Boundary Layers in Extragalactic Jets

D.S. De Young

Fractal Properties of Extragalactic Jets: Evidence of Turbulence? . ...463

G. Bodo, S. Massaglia, L. Feretti, et al.

ISM / Radio Material Interaction Modeling .................. 465

P. Ferruit, L. Binette E. Pecontal

Numerical Simulations of Extragalactic Jets

in an Inhomogeneous Environment

S. Higgins, T. O'Brien, J. Dunlop

Shock Excitation of Emission Lines and their Relation

to GPS Sources

G.V. Bicknell, M.A. Dopita, C.P. O'Dea

Dynamical Models Emission-Line Gas in Radio Galaxies

A.M. Koekemoer, G.V. Bicknell

Shock Excitation of Emission Lines in Radio Galaxies

A.M. Koekemoer, G.V. Bicknell 
Comparative Study of Extragalactic Radio Sources

from "Double Jet" models

H. Dole, H. Sol, L. Vicente

Distortion Effects in BL Lac Radio Jets

H. Sol, S. Appl, L. Vicente

Describing the Asymmetries in Extragalactic Radio Sources

S. Ryś

Analytical Modelling of Linear Size Evolution

of Powerful Radio Sources

K.T. Chyży

How Radio Sources Stay Young

J.A. Eilek

A Cocoon Transparency and the 3C 345 Low Frequency Variability .. 485 L.I. Matveenko

Simulation of the Variable Multifrequency Radio Emission and

Structure for the Quasar 2145+067

Y.Y. Kovalev

The Spectral and Spatial Decomposition

of Extragalactic Radio Sources

L. Rudnick, D.M. Katz-Stone

Discussion

\section{Surveys of Radio Sources}

The Westerbork Northern Sky Survey (Invited)

A.G. de Bruyn

First Results from the VLA FIRST Survey (Invited)

R.H. Becker, M.D. Gregg, D.J. Helfand, et al.

The NRAO VLA D-Array Sky Survey (NVSS) (Invited) ..........503

W.D. Cotton, J.J. Condon, Q.F. Yin, et al.

The Parkes-MIT-NRAO Radio Surveys (Invited) ..............507

A.E. Wright, N.J. Tasker, A. Savage, A.E. Vaughan

The Distant DRAGNs Survey ............................513

J.D.B. Law-Green

2-Jy Sample of Southern Radio Sources ......................515

R. Morganti, C.N. Tadhunter, R. Dickson, et al.

The MRC 1-Jy Sample of Radio Galaxies .................... 517

V.K. Kapahi, P.J. McCarthy, R.M. Athreya, et al.

The B3-VLA Sample .

M. Vigotti, S.G. Djorgovski, L. Gregorini, et al. 
A Deep $20 \mathrm{~cm}$ Radio Mosaic of the ESO Key-project Galaxy

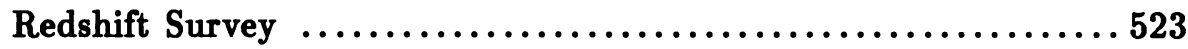

I. Prandoni, L. Gregorini, P. Parma, et al.

Radio Observations of the Marano Field

Sub-MJy Source Counts and Spectral Index Studies

C. Gruppioni, P. Parma, H.R. de Ruiter, G. Zamorani

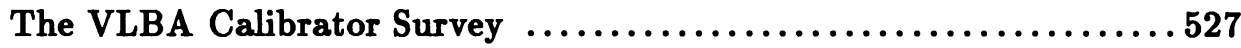

A.J. Beasley, V. Dhawan, E.B. Fomalont, et al.

The VSOP Mission for Extragalactic Radio Sources

H. Hirabayashi

Imaging of Extragalactic Radio Sources with

the VSOP Space VLBI Mission

D.W. Murphy

Discussion

\section{Cosmological Implications}

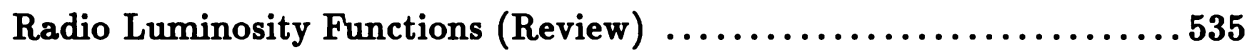
J.J. Condon

A nuclear Luminosity Function for Seyferts

E.J.A. Meurs

VLA Observations of the Cambridge-Cambridge Rosat Survey ......543

P. Ciliegi, M. Elvis, B.J. Wilkes, et al.

On the Cosmological Evolution of Radio Quiet

and Radio Loud Quasars ............................. 545

J. Machalski

Space Distribution of Radio Source Populations (Review)

J.V. Wall

Space Densities for Powerful Radio Sources

in the Light of Unification

C.A. Jackson, J.V. Wall

The Radio Sky at Micro-Jy Levels (Invited)

E.B. Fomalont

Redshift Distribution and Nature of $\mu$-Jy Radio Sources

F. Hammer, O. Le Fèvre, D. Crampton, et al.

The Space Density of High-Redshift Quasars

P.A. Shaver, J.V. Wall, K.I. Kellermann, et al.

Cosmological Size Evolution of Extragalactic Radio Sources (Invited) 563 A.K. Singal

New Results from Complete Samples of Faint

Radio Galaxies and Quasars

K.M. Blundell, S. Rawlings, S.A. Eales, M. Lacy 
Spectral Index-Redshift Relation for Radio Galaxies and Quasars ....569 R.D. Dagkesamanskii

High Redshift Radio Galaxies (Review) 571

K. Meisenheimer, H. Hippelein, M. Neeser

HST and KECK Observations of

High Redshift Radio Galaxies (Invited)

W.J.M. van Breugel

High Redshift Milli-Jansky Radio Galaxies

J. Dunlop, J. Peacock, R. Windhorst, et al.

Distant Radio Galaxies: the Strong Link

between the Radio and Optical Emission

H. Röttgering

The R-Band Hubble Diagram for GPS Galaxies

I. Snellen, M. Bremer, R. Schilizzi, et al.

Stellar Population Models of Distant Radio Galaxies

D. Villani, S. di Serego Alighieri

The RATAN-600 - VLA - 6m Russian telescope:

Early Universe Project

Y.N. Parijskij, N.S. Soboleva, W.M. Goss, et al.

Faint Radio Sources and the Cosmic Microwave Background

E.A. Richards

FSRQ and the Gamma-Ray Background

T. di Girolamo, A. Comastri, G. Setti

Discussion ......................................... 597

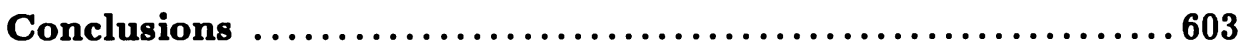

L. Woltjer

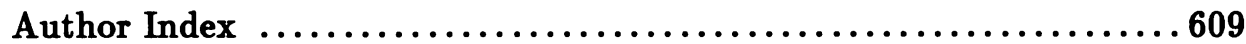

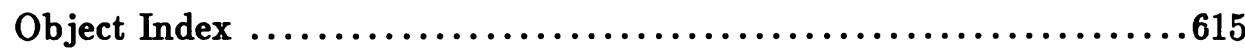

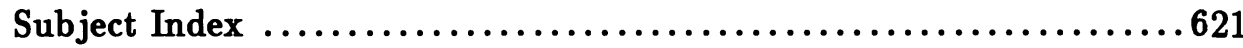

